\title{
Perinatale Betreuung an der Grenze der Lebensfähigkeit zwischen 22 und 26 vollendeten Schwangerschaftswochen
}

\author{
Die hier vorgestellten überarbeiteten Empfehlungen wurden durch eine Kommis- \\ sion erfahrener Spezialisten ausgearbeitet und von den wichtigsten Fachgesell- \\ schaften und -verbänden genehmigt. Auch die Zentrale Ethikkommission der SAMW \\ unterstützt die Empfehlungen, deren wichtigste Neuerungen hier zusammengefasst \\ sind.*
}

\section{T. M. Berger ${ }^{a}$ \\ (red. Verantwortung), \\ V. Bernet ${ }^{a}$, \\ S. El Alama d, J.-C. Fauchèrea, \\ I. Hösli ${ }^{b}$, O. Irion ${ }^{b}$, C. Kind ${ }^{a}$, \\ B. Latal', M. Nellea, \\ R.E. Pfister ${ }^{a}$, D. Surbek ${ }^{b}$, \\ A.C. Truttmann ${ }^{a}$, J. Wisser ${ }^{b}$, \\ R. Zimmermann ${ }^{b}$ (alph.)}

Arbeitsgruppe folgender Gesellschaften:

a Schweizerische Gesellschaft für Neonatologie

b Schweizerische Gesellschaft für Gynäkologie und Geburtshilfe und Schweizerische Akademie für fetomaternale Medizin

c Schweizerische Gesellschaft für Entwicklungspädiatrie

d Schweizerischer Hebammenverband

* Die Gesamtversion der Empfehlungen wurde im Swiss Medical Weekly auf Englisch publiziert [25] (www.smw.ch)

** Die Literaturangaben finden sich unter www.saez.ch $\rightarrow$ Aktuelle Nummer oder $\rightarrow$ Archiv $\rightarrow 2012 \rightarrow 4$.

Korrespondenz:

Prof. Dr. T. M. Berger Neonatologische und Pädiatrische Intensivpflegestation Kinderspital Luzern CH-6000 Luzern

Tel. 0412053285

thomas.berger[at]ksl.ch

\section{Einleitung}

Die ersten Empfehlungen zur Betreuung von Frühgeborenen an der Grenze der Lebensfähigkeit in der Schweiz wurden im Jahre 2002 veröffentlicht [1]**. Als Grundlage dienten damals unter anderem Empfehlungen europäischer [2, 3] und kanadischer Fachgruppen [4] sowie die relevanten medizinisch-ethischen Richtlinien der Schweizerischen Akademie der Medizinischen Wissenschaften (SAMW) [5, 6]. Revidierte Empfehlungen aus Nordamerika und Europa [7-11], neue Empfehlungen aus weiteren Ländern [12-17] und neue Daten zu Mortalität und Morbidität [18-22], insbesondere auch aus der Schweiz [23, 24], haben Anlass dazu gegeben, die Empfehlungen für die Schweiz zu überarbeiten.

Nationale Empfehlungen sind notwendig, da ethische Entscheidungsfindungen nicht alleine auf allseits anerkannten ethischen Prinzipien basieren, sondern durch gesellschaftliche, ökonomische und legale Überlegungen mit beeinflusst werden. Qualitativ gute Daten zu erzielbaren Langzeitresultaten sind ebenfalls wichtig und die verfügbaren Studien belegen, dass die Resultate zwischen verschiedenen Ländern erheblich variieren [18-24]. Aus diesen Gründen ist davon auszugehen, dass Empfehlungen aus anderen Ländern nicht ohne weiteres auf die Schweiz übertragbar sind. Eine Akzeptanz der Empfehlungen ist aber anzustreben, um die Behandlungsvariabilität zwischen den Zentren, die Schwangere mit hohem Risiko für eine Frühgeburt und Frühgeborene an der Grenze der Lebensfähigkeit in der Schweiz betreuen, zu minimieren.

Die vorliegenden Empfehlungen wurden durch eine Kommission von erfahrenen Spezialisten ausgearbeitet. Die vorgeschlagenen Änderungs- und Ergänzungsvorschläge wurden an fünf Workshops in den Jahren 2009 und 2010 ausführlich diskutiert. Die neuen Empfehlungen wurden von allen Mitgliedern der Arbeitsgruppe gutgeheissen und 2011 von der Schweizerischen Gesellschaft für Gynäkologie und Geburtshilfe (SGGG), der Akademie für fetomaternale Medizin (AFMM), dem Schweizerischen Hebammenverband (SHV), der Schweizerischen Gesellschaft für Pädiatrie (SGP), der Schweizerischen Gesellschaft für Neonatologie (SGN) und der Schweizerischen Gesellschaft für Entwicklungspädiatrie (SGEP) genehmigt. Wie bereits im Jahre 2002 unterstützt die Zentrale Ethikkommission (ZEK) der SAMW die neuen Empfehlungen. Die Empfehlungen wurden im Swiss Medical Weekly in englischer Sprache publiziert [25] und können über www.smw.ch oder via PubMed online gelesen oder als PDF heruntergeladen werden (open access). An dieser Stelle sollen lediglich die wesentlichen Neuerungen hervorgehoben und eine deutsche Version der Zusammenfassung präsentiert werden.

\section{Was ist neu?}

Im Vergleich zur Version aus dem Jahre 2002 enthalten die neuen Empfehlungen evidenzbasierte Informationen über geburtsmedizinische Massnahmen, insbesondere zur fetalen Lungenreifung und zum Stellenwert der Sectio-Entbindung. In den alten Empfehlungen basierten die vorgeschlagenen Vorgehensweisen in erster Linie auf dem Gestationsalter. In den revidierten Empfehlungen werden neben dem Gestationsalter zusätzliche, pränatal eruierbare Faktoren, die einen erheblichen Einfluss auf die Prognose haben, mitberücksichtigt (Tabelle 1). Die Grauzone wird enger definiert, ihre Grenzen aber werden weniger absolut gesehen, um eine individualisierte Vorgehensweise an der Grenze der Lebensfähigkeit zu ermöglichen. Die Bedeutung der Kommunikation wird erneut betont und die Rolle der Eltern bei der Entscheidungsfindung wird detaillierter dargestellt. 


\section{Zusammenfassung}

Diese Empfehlungen beziehen sich auf die perinatale Betreuung von Schwangeren mit hohem Risiko für eine Frühgeburt und von Frühgeborenen an der Grenze der Lebensfähigkeit (Gestationsalter zwischen 22 und 26 vollendeten Schwangerschaftswochen SSW). Sie richten sich an Ärzte, Hebammen, Pflegefachpersonen und Mitglieder anderer Berufsgruppen, die bei dieser Betreuung mitwirken (zur sprachlichen Vereinfachung wird im folgenden Text bei Personenangaben nur die männliche Form gebraucht).

Die perinatale Betreuung von Schwangeren mit hohem Risiko für eine Frühgeburt und Frühgeborenen an der Grenze der Lebensfähigkeit (22-26 vollendete Schwangerschaftswochen) muss multidisziplinär durch ein erfahrenes perinatologisches Team erfolgen. Dabei ist zu berücksichtigen, dass sowohl die eingeschränkte Präzision der Bestimmung des Gesta-
Die für die Schwangere und das Frühgeborene zu treffenden Entscheidungen sind komplex und von weitreichender Bedeutung. Ein möglichst umfassendes und einfühlsames Pränatalgespräch, das in verständlicher Sprache von Neonatologen und Geburtshelfern gemeinsam mit den Eltern geführt wird, ist wichtig, um eine Vertrauensbasis aufzubauen und Unterstützung zu bieten. Die Entscheidungen werden in einem kontinuierlichen Dialog zwischen allen Beteiligten (Ärzte, Hebammen, Pflegepersonal und Eltern) erarbeitet und haben zum Ziel, die Massnahmen zu ergreifen, die im Interesse des Kindes und der Schwangeren als die besten erachtet werden.

Die Kenntnis aktueller nach Gestationsalter abgestufter Mortalitäts- und Morbiditätsstatistiken sowie deren Beeinflussung durch pränatal eruierbare $\mathrm{Zu}$ satzfaktoren (fetales Gewicht, Geschlecht, Lungenreifungsinduktion erfolgt oder nicht, Mehrlingsschwan-

\section{«Die Entscheidungen werden in einem kontinuierlichen Dialog zwischen allen Beteiligten (Ärzte, Hebammen, Pflegepersonal und Eltern) erarbeitet.»}

tionsalters und des geschätzten fetalen Gewichtes als auch die biologische Variabilität das Vorgehen im individuellen Fall entscheidend beeinflussen können. gerschaft oder Einlingsschwangerschaft) und die Anwendung anerkannter ethischer Grundprinzipien bilden dabei die Basis für ein verantwortungsvolles

\footnotetext{
Tabelle 1

Einfluss von pränatal eruierbaren Zusatzfaktoren (Geschlecht, Lungenreifungsinduktion (LRI), Einling/Mehrling, Geburtsgewicht) auf die Wahrscheinlichkeit eines Überlebens ohne schwere oder sehr schwere Behinderung (nach Tyson et al. [20]).

\begin{tabular}{|l|c|c|c|c|c|c|c|}
\hline $\begin{array}{l}\text { Gestationsalter } \\
\text { (SSW) }\end{array}$ & Geschlecht & $\begin{array}{c}\text { Geburts- } \\
\text { gewicht }\end{array}$ & Einling & LRI & $\begin{array}{c}\text { Überlebens- } \\
\text { rate }{ }^{1}\end{array}$ & $\begin{array}{c}\text { Überleben ohne } \\
\text { sehr schwere } \\
\text { Behinderung }\end{array}$ & $\begin{array}{c}\text { Überleben ohne } \\
\text { schwere } \\
\text { Behinderung }\end{array}$ \\
\hline $240 / 7-246 / 7$ & m & $600 \mathrm{~g}$ & nein & nein & $27 \%$ & $15 \%$ & $7 \%$ \\
\hline $240 / 7-246 / 7$ & w & $600 \mathrm{~g}$ & nein & nein & $36 \%$ & $24 \%$ & $14 \%$ \\
\hline $240 / 7-246 / 7$ & w & $800 \mathrm{~g}$ & nein & nein & $63 \%$ & $48 \%$ & $34 \%$ \\
\hline $240 / 7-246 / 7$ & w & $800 \mathrm{~g}$ & ja & nein & $67 \%$ & $53 \%$ & $39 \%$ \\
\hline $240 / 7-246 / 7$ & w & $800 \mathrm{~g}$ & ja & ja & $82 \%$ & $71 \%$ & $57 \%$ \\
\hline
\end{tabular}

1 in Prozent aller Lebendgeborenen $(n=4446)$

2 sehr schwere Behinderung (profound impairment) im Alter von18-22 Monaten:

- Bayley score $<50$ (nicht testbar)

- level 5 für GMF (gross motor function)

3 schwere Behinderung (severe impairment) im Alter von18-22 Monaten:

- PDI und/oder MDI $\leq 70$

- mittelschwere bis schwere CP

- bilaterale Blindheit

- bilaterale Schwerhörigkeit, die Hörgeräte erforderlich macht

Diese Schätzungen basieren auf standardisierten Untersuchungen von ehemaligen Frühgeborenen im Alter von 18-22 Monaten, die zwischen 1998 und 2003 in einem Neonatal Research Network Zentrum der NICHD mit einem Gestationsalter von 22-25 SSW und einem Geburtsgewicht zwischen 401 und 1000 g geboren wurden. Outborns und Frühgeborene mit schweren angeborenen Fehlbildungen wurden ausgeschlossen.
} 


\section{Abbildung 1}

Einfluss positiver Zusatzfaktoren und Rolle der elterlichen Autorität beim Abwägen der Therapieoptionen für Frühgeborene mit einem Gestationsalter von 23 0/7-23 6/7 Schwangerschaftswochen

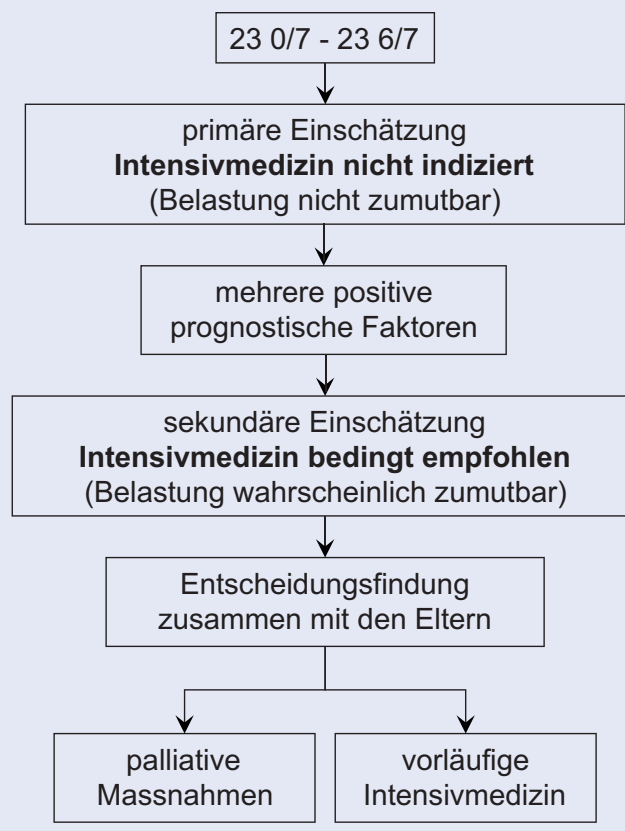

Vorgehen. Die Kommunikation zwischen den beteiligten Entscheidungsträgern spielt eine zentrale Rolle.

Die Mitglieder der interdisziplinären Arbeitsgruppe schlagen vor, dass sich die Betreuung von Frühgeborenen mit einem Gestationsalter $<24$ SSW in der Regel auf Palliativmassnahmen beschränken soll. Geburtsmedizinische Massnahmen (z.B. eine SectioEntbindung) sind aus fetaler Indikation in der Regel nicht indiziert. Sie können kombiniert mit einer intensivmedizinischen Betreuung des Frühgeborenen in ausgewählten Fällen sinnvoll sein, z. B. nach der vollendeten 23. SSW, wenn mehrere der bereits erwähnten pränatal erkennbaren Zusatzfaktoren die Prognose günstig beeinflussen oder zuvor eingehend aufgeklärte Eltern auf dem Einsatz lebenserhaltender Massnahmen bestehen (Abb. 1).

Bei Frühgeborenen mit einem Gestationsalter von 24 0/7-24 6/7 SSW kann die Beurteilung schwierig sein, ob das durch die geburtsmedizinischen Massnahmen (z. B. Sectio-Entbindung) und die intensivmedizinische Behandlung des Frühgeborenen zugefügte Leiden angesichts der begrenzten Erfolgschancen zumutbar ist. Die Konstellation der vorliegenden prognostisch relevanten Zusatzfaktoren kann in dieser Situation für die gemeinsame Entscheidfindung mit den Eltern richtungweisend sein (Abb. 2).

Bei Frühgeborenen mit einem Gestationsalter zwischen $250 / 7$ bis 25 6/7 SSW sind fetale Überwachung, geburtsmedizinische Massnahmen und eine

\section{Abbildung 2}

Einfluss positiver resp. negativer Zusatzfaktoren und Rolle der elterlichen Autorität beim Abwägen der Therapieoptionen für Frühgeborene mit einem Gestationsalter von 24 0/7-24 6/7 Schwangerschaftswochen.

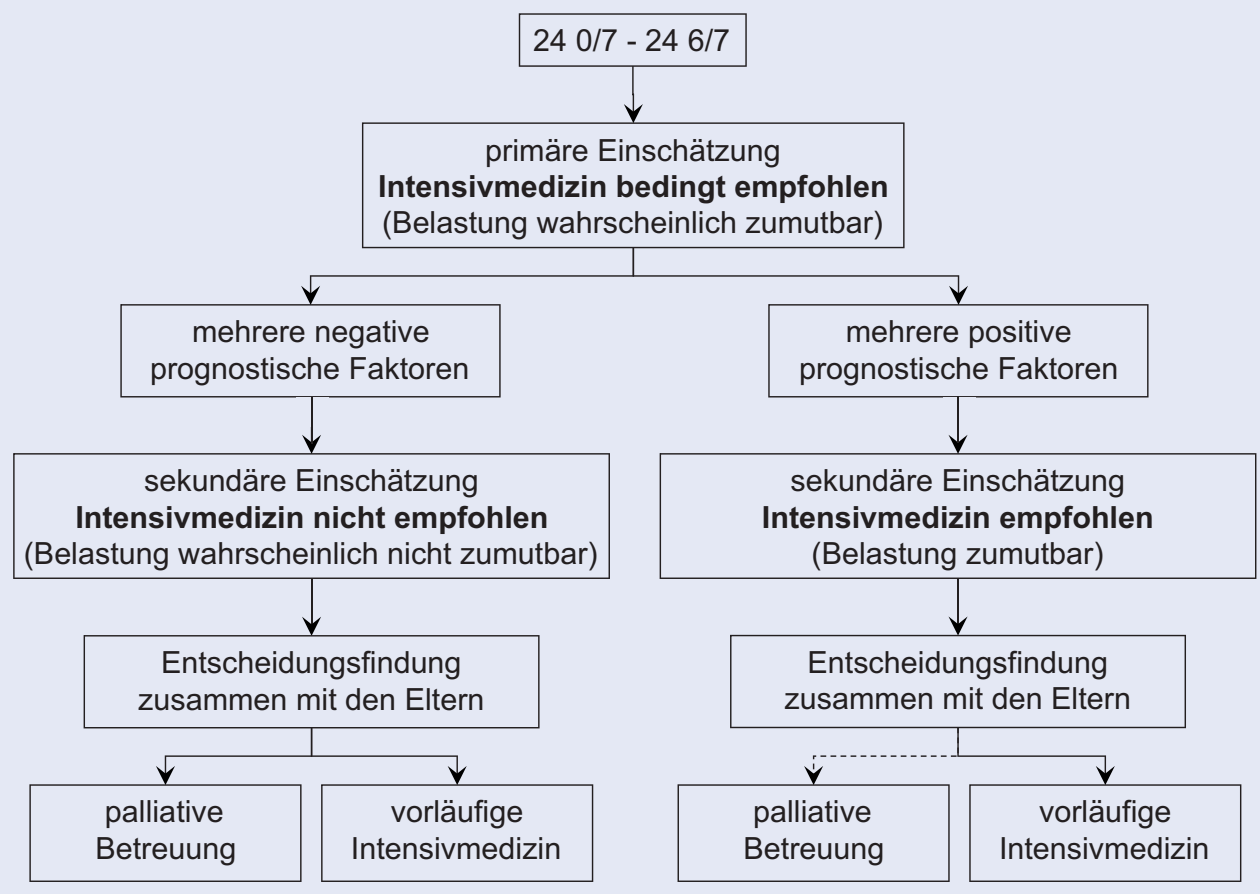




\section{Abbildung 3}

Einfluss negativer Zusatzfaktoren und Rolle der elterlichen Autorität beim Abwägen der Therapieoptionen für Frühgeborene mit einem Gestationsalter von 25 0/7-25 6/7 Schwangerschaftswochen.

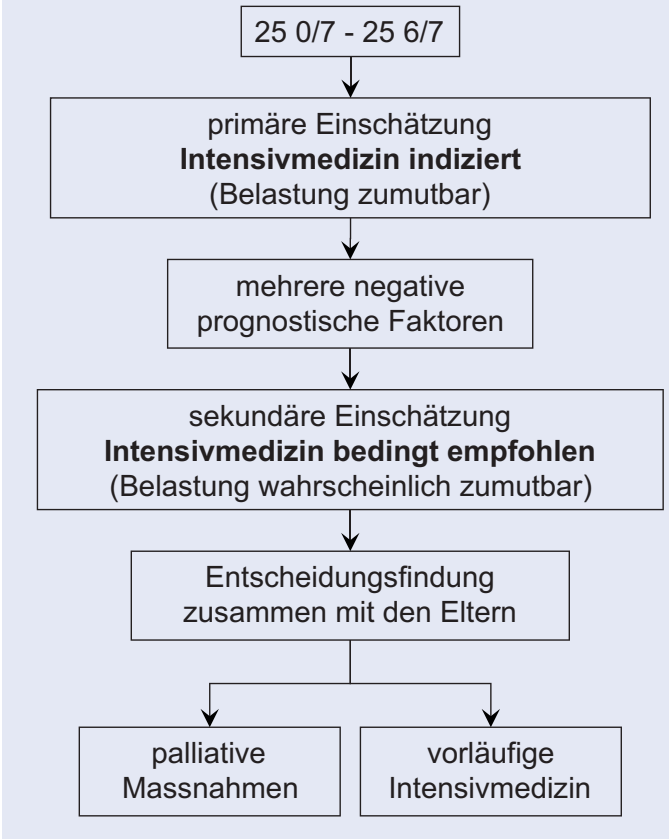

Ein erfahrenes Neonatologie-Team sollte bei allen Geburten, die nach 23 0/7 SSW stattfinden, involviert werden, um zusammen mit den Eltern individuell zu entscheiden, ob der Einsatz intensivmedizinischer Massnahmen gerechtfertigt erscheint oder nur Palliativmassnahmen zur Anwendung kommen sollen (sog. primary non-intervention). Aus diesem Grunde müssen alle Schwangeren mit drohender Frühgeburt oder vorzeitigem Blasensprung spätestens ab der 23. 0/7 SSW in ein perinatologisches Zentrum mit angeschlossener neonatologischer Intensivstation verlegt werden. In unklaren Fällen kann es sinnvoll sein, eine intensivmedizinische Betreuung $\mathrm{zu}$ beginnen und das Frühgeborene in die Neugeborenen-Intensivstation zu verlegen (provisional intensive care). Der klinische Verlauf und weitere Gespräche mit den Eltern können dann Klarheit darüber verschaffen, ob die intensivmedizinischen Massnahmen fortgesetzt oder abgebrochen werden sollen.

Solange aufgrund engmaschiger Beurteilungen berechtigte Hoffnung darauf besteht, dass das Frühgeborene überleben kann und die begonnene Intensivtherapie für das Kind zumutbar ist, werden die ergriffenen Massnahmen fortgesetzt. Müssen das betreuende Team und die Eltern jedoch erkennen, dass das durch die Therapie zugemutete Leiden angesichts der sehr ungünstig gewordenen Prognose unverhältnismässig geworden ist, verlieren die intensivmedizinischen Massnahmen ihren Sinn, und andere Aspekte der

\section{«Müssen das betreuende Team und die Eltern jedoch erkennen, dass}

das durch die Therapie zugemutete Leiden angesichts der sehr ungüns-

\section{tig gewordenen Prognose unverhältnismässig geworden ist, verlieren die intensivmedizinischen Massnahmen ihren Sinn.»}

intensivmedizinische Betreuung des Frühgeborenen in der Regel indiziert. Liegen allerdings mehrere prognostisch ungünstige Zusatzfaktoren vor, kann bei der gemeinsamen Entscheidfindung mit den Eltern eine rein palliative Betreuung des Frühgeborenen in Betracht gezogen werden (Abb. 3).
Betreuung (Linderung von Schmerzen und Leiden) werden prioritär (redirection of care). Wird auf lebenserhaltende Interventionen verzichtet (primär oder sekundär), sollte alles getan werden, um dem Kind ein menschenwürdiges Sterben zu ermöglichen (comfort care) und die Eltern in der Sterbebegleitung zu unterstützen. 


\section{Literatur}

1 Berger TM, Büttiker V, Fauchère JC, Holzgreve W, Kind C, Largo R, et al. Empfehlungen zur Betreuung von Frühgeborenen an der Grenze der Lebensfähigkeit (Gestationsalter 22-26 SSW). Schweiz Ärztezeitung. 2002;83(29/30):1589-95.

2 Gee H, Dunn P, for the BAPM Executive Committee. Fetuses and newborn infants at the threshold of viability. A framework for practice. www. bapm-london.org/publications.htm. Published 2000

3 Pohlandt F. Frühgeburt an der Grenze der Lebensfähigkeit des Kindes. PerinatalMedizin. 1998; 10:99-101.

4 Canadian Paediatric Society, Fetus and Newborn Committee, and Society of Obstetricians and Gynaecologists of Canada, Maternal-Fetal Medicine Committee. Management of the woman with threatened birth of an infant of extremely low gestational age. CMJA. 1994;151(5):547-53.

5 SAMW. Medizinisch-ethische Richtlinien für die ärztliche Betreuung sterbender und zerebral schwerst geschädigter Patienten. Schweiz Ärztezeitung. 1995;76:1223-5.

6 SAMW. Medizinisch-ethische Richtlinien zu Grenzfragen der Intensivmedizin. Schweiz Ärztezeitung. 1999;80:2134-8

7 Kattwinkel J, Perlman JM, Aziz K, Colby C, Fairchild K, Gallagher J, et al. Neonatal resuscitation: 2010 American Heart Association Guidelines for Cardiopulmonary Resuscitation and Emergency Cardiovascular Care. Pediatrics. 2010;126(5):e1400-13.

8 MacDonald H. Perinatal care at the threshold of viability. Pediatrics. 2002;110(5):1024-7.

9 Pohlandt F. [Premature birth at the boundary of infant viability]. Z Geburtshilfe Neonatol. 2008;212(3):109-13.

10 Richmond S, Wyllie J. European Resuscitation Council Guidelines for Resuscitation 2010 Section 7. Resuscitation of babies at birth. Resuscitation. 2010;81(10):1389-99.

11 Wilkinson AR, Ahluwalia J, Cole A, Crawford D, Fyle J, Gordon A, et al. Management of babies born extremely preterm at less than 26 weeks of gestation: a framework for clinical practice at the time of birth. Arch Dis Child Fetal Neonatal Ed. 2009;94(1):F2-5.

12 Lui K, Bajuk B, Foster K, Gaston A, Kent A, Sinn J, et al. Perinatal care at the borderlines of viability: a consensus statement based on a NSW and ACT consensus workshop. Med J Aust. 2006;185(9): 495-500.

13 Miljeteig I, Markestad T, Norheim OF. Physicians' use of guidelines and attitudes to withholding and withdrawing treatment for extremely premature neonates in Norway. Acta Paediatr. 2007;96(6):825-9.
14 Moriette G, Rameix S, Azria E, Fournie A, Andrini P, Caeymaex L, et al. [Very premature births: Dilemmas and management. Part 1. Outcome of infants born before 28 weeks of postmenstrual age, and definition of a gray zone]. Arch Pediatr. 2010;17(5):518-26.

15 Moriette G, Rameix S, Azria E, Fournie A, Andrini P, Caeymaex L, et al. [Very premature births: Dilemmas and management. Second part: Ethical aspects and recommendations]. Arch Pediatr. 2010;17(5):527-39.

16 Pignotti MS, Scarselli G, Barberi I, Barni M, Bevilacqua G, Branconi F, et al. Perinatal care at an extremely low gestational age (22-25 weeks). An Italian approach: the «Carta di Firenze». Arch Dis Child Fetal Neonatal Ed. 2007;92(6):F515-6.

17 Verloove-Vanhorick SP. Management of the neonate at the limits of viability: the Dutch viewpoint. BJOG. 2006;113 Suppl 3:13-6.

18 Doyle LW, Roberts G, Anderson PJ. Outcomes at age 2 years of infants $<28$ weeks $>$ gestational age born in Victoria in 2005. J Pediatr. 2010;156(1):49-53 e41.

19 Fellman V, Hellstrom-Westas L, Norman M, Westgren M, Kallen K, Lagercrantz H, et al. One-year survival of extremely preterm infants after active perinatal care in Sweden. JAMA. 2009;301(21):2225-33.

20 Tyson JE, Parikh NA, Langer J, Green C, Higgins RD. Intensive care for extreme prematurity-moving beyond gestational age. N Engl J Med. 2008; 358(16):1672-81.

21 Mercier CE, Dunn MS, Ferrelli KR, Howard DB, Soll RF. Neurodevelopmental Outcome of Extremely Low Birth Weight Infants from the Vermont Oxford Network: 1998-2003. Neonatology. 2009;97(4) 329-38.

22 Stoll BJ, Hansen NI, Bell EF, Shankaran S, Laptook AR, Walsh MC, et al. Neonatal outcomes of extremely preterm infants from the NICHD Neonatal Research Network. Pediatrics. 2010;126(3):443-56.

23 Fischer N, Steurer MA, Adams M, Berger TM. Survival rates of extremely preterm infants (gestational age $<26$ weeks) in Switzerland: impact of the Swiss guidelines for the care of infants born at the limit of viability. Arch Dis Child Fetal Neonatal Ed. 2009;94(6):F407-13

24 Bajwa N, Berner M, Worley S, Pfister R, Swiss Neonatal Network. Population-based age stratified morbidities of premature infants in Switzerland. Swiss Med Wkly. 2011;141:w13212.

25 Berger TM, Bernet V, El Alama S, Fauchere JC, Hösli I, Irion $\mathrm{O}$, et al. Perinatal care at the limit of viability between 22 and 26 completed weeks of gestation in Switzerland. Swiss Med Wkly. 2011;141:w13280. 